\title{
Effects of mrpigG on Development and Secondary Metabolism of Monascus ruber M7
}

\author{
Li Li ${ }^{1,2}$ and Fusheng Chen $1,2, * \mathbb{D}$ \\ 1 Hubei International Scientific and Technological Cooperation Base of Traditional Fermented Foods, \\ Huazhong Agricultural University, Wuhan 430070, China; lili1991@webmail.hzau.edu.cn \\ 2 College of Food Science and Technology, Huazhong Agricultural University, Wuhan 430070, China \\ * Correspondence: chenfs@mail.hzau.edu.cn
}

Received: 29 July 2020; Accepted: 26 August 2020; Published: 29 August 2020

\begin{abstract}
Monascus pigments (MPs) have been used as food colorants for several centuries in Asian countries and are now used throughout the world via Asian catering. The MP biosynthetic pathway has been well-illustrated, but the functions of a few genes, including mrpigG, in the MP gene cluster are still unclear. In the current study, in order to investigate the function of mrpigG in M. ruber M7, gene deletion ( $\Delta$ mrpigG), complementation ( $\Delta$ mrpigG::mrpigG) and overexpression (M7::PtrpC-mrpigG) mutants were successfully obtained. The morphologies and biomasses, as well as the MP and citrinin production, of these mutants were analyzed. The results revealed that the disruption, complementation and overexpression of mrpigG showed no apparent defects in morphology, biomass or citrinin production (except MP production) in $\Delta$ mrpigG compared with M. ruber M7. Although the MP profiles of $\Delta$ mrpigG and M. ruber M7 were almost the same-with both having four yellow pigments, two orange pigments (OPs) and two red pigments (RPs) — their yields were decreased in $\Delta$ mrpigG to a certain extent. Particularly, the content of rubropunctatin (an OP) and its derivative rubropunctamine (an RP) in $\Delta$ mrpigG, both of which have a five-carbon side chain, accounted for $57.7 \%$, and $22.3 \%$ of those in M. ruber M7. On the other hand, monascorubrin (an OP) and its derivative monascorubramine (an RP), both of which have a seven-carbon side chain, were increased by 1.15 and 2.55 times, respectively, in $\Delta$ mrpigG compared with $M$. ruber M7. These results suggest that the MrPigG protein may preferentially catalyze the biosynthesis of MPs with a five-carbon side chain.
\end{abstract}

Keywords: Monascus ruber; mrpigG; development; secondary metabolism

\section{Introduction}

Monascus spp., a type of medicinal and edible filamentous fungus, have been used to produce fermented foods and medicine in Asian countries, such as China, Japan and the Korean Peninsula, for nearly 2000 years [1,2]. At present, their fermented products, such as red fermented rice (RFR), also called red yeast rice, Anka, Hongqu, red Koji, and red mold rice, are widely used as food additives and nutraceutical supplements worldwide, owing to their production of beneficial secondary metabolites (SMs), mainly including Monascus pigments (MPs), monacolin K (MK) and $\gamma$-amino butyric acid [1,3,4], even though some strains of Monascus spp. may produce citrinin (CIT), a kind of nephrotoxic mycotoxin [5,6].

MPs are a complex mixture of compounds with a common azaphilone skeleton [7], and more than $110 \mathrm{MP}$ components, most of which possess a five- or seven-carbon side chain, have been identified to date [8]. Despite their large-scale utilization and research, the biosynthesis of MPs remains inadequate to a certain extent. In the last 10 years, a unifying picture of the biosynthesis of MPs has emerged as a result of genome sequencing and the functional analysis of MPs' biosynthetic gene clusters. In 2017, 
Chen et al. comprehensively proposed the biosynthetic pathway of MPs in M. ruber M7 [9]. Although the structures of MPs are extremely diverse, their biosynthesis employs a unitary trunk pathway that ushers intermediates towards the classical yellow (monascine, ankaflavin), orange (rubropunctatin, monascorubrin) and red (rubropunctamine, monascorubramine) pigments, and features a variety of shunt pathways branching off from the trunk pathway at highly reactive node compounds [9]. The functions of many genes in the MP gene clusters of $M$. ruber M7 have been investigated by gene modification. However, there are a few genes in the MP gene cluster, such as mpigG, mpigH, and mpigI, which have not been examined to date. In 2013, Balakrishnan et al. found that the mppD deletion in M. purpureus KACC (mrpigG in M. ruber M7) resulted in a significant decrease in the production of four MPs and speculated that MppD might be an obligate accessory protein involved in the product formation of a precursor of MPs, but no experimental data were provided [10]. In 2019, Chen et al. also guessed that MrPigG may contribute to the release of the highly reactive intermediate of MrPigA [8].

In this study, we first cloned the mrpigG gene from $M$. ruber M7, which encodes a protein sharing $93.01 \%$ amino acid sequence similarity with the amino oxidase/esterase of M. pilosus (Genbank: AGN71609.1) and 69.26\% similarity with serine hydrolase FSH of Penicillium occitanis (Genbank: PCH03976.1). Subsequently, we constructed mrpigG deletion $(\Delta m r p i g G)$, complementation ( $\Delta$ mrpigG::mrpigG) and overexpression (M7::PtrpC-mrpigG) mutants of M. ruber M7, and analyzed their morphologies, biomasses, as well as their MP and CIT production. The results revealed that the mrpigG mutants have inconspicuous effects on their morphologies, growth and MP and citrinin production, except for MPs production in $\triangle m r p i g G$, which implies that MrPigG may prefer to biosynthesize MP compounds with a five-carbon side chain, compared to ones with a seven-carbon side chain. To our knowledge, this is the first report accounting for the mechanism used by genes in the MP gene cluster to control the length of the side chains of MPs.

\section{Materials and Methods}

\subsection{Fungal Strains, Culture Media and Growth Conditions}

M. ruber M7 (CCAM 070120, Culture Collection of State Key Laboratory of Agricultural Microbiology, Wuhan, China), which can produce MPs and CIT, but no MK, was used as a DNA donor and for transformation [11]. All strains used in this study are described in Table 1. Potato dextrose agar (PDA), malt extract agar (MA), Czapek yeast extract agar (CYA) and 25\% glycerol nitrate agar (G25N) were utilized for morphological characterization [12,13]. PDA medium was used for the analyses of MPs and CIT production. G418 (Sigma-Aldrich, Shanghai, China) or hygromycin B (Sigma-Aldrich, Shanghai, China) was added to the media for transformant selection [14]. All strains were maintained on PDA slants at $28^{\circ} \mathrm{C}$.

Table 1. Monascus ruber strains constructed and used in this study.

\begin{tabular}{cccc}
\hline Strain & Parent & Genotype & Source \\
\hline M7 & M7 & Wild-type & Red fermented rice [11] \\
\hline$\Delta$ mrpigG & M7 & $\Delta$ mrpigG::hph & This study \\
\hline$\Delta$ mrpigG:: mrpigG & $\Delta$ mrpigG & $\Delta$ mrpigG::mrpigG-neo & This study \\
\hline M7::PtrpC-mrpigG & M7 & M7::PtrpC-mrpigG-neo & This study \\
\hline
\end{tabular}

\subsection{Cloning and Analysis of the mrpigG Gene}

A pair of primers, pigG F1-pigG R1 (Table 2), were designed to amplify the mrpigG gene, using Oligo 6 software (http://www.oligo.net/). PCR was carried out to amplify the mrpigG gene from the genome of M. ruber M7. Amino acid sequences encoded by mrpig $G$ were predicted using Soft Berry's FGENESH program (http://www.softberry.com), and the MrPigG functional regions were analyzed 
using the Pfam 33.1 program (http://pfam.xfam.org/). Homology of the deduced amino acid sequence was analyzed using the BLASTP program on the NCBI website (http://blast.ncbi.nlm.nih.gov/Blast.cgi).

Table 2. Primers used in this study.

\begin{tabular}{|c|c|c|}
\hline Names & Sequences $\left(5^{\prime} \rightarrow 3^{\prime}\right)$ & Descriptions \\
\hline pigG F1 & GATCTGCCAGAAATACTAGA & \multirow{2}{*}{$\begin{array}{l}\text { For amplification of the } 1236 \mathrm{bp} \text { of the whole } \\
\text { mrpigG gene }\end{array}$} \\
\hline pigG R1 & TCGGCGAAGCAGAGGCGGAA & \\
\hline pigG d5F & CGTCCCCCTTCTGCCCAAGA & \multirow{2}{*}{$\begin{array}{l}\text { For amplification of the } 884 \mathrm{bp} \text { of } 5^{\prime} \text { flanking } \\
\text { regions of the mrpigG gene }\end{array}$} \\
\hline pigG d5R & CAATATCATCTTCTGTCGAC CCGAACTCCTTGTAGACCGA & \\
\hline hph F & GTCGACAGAAGATGATATTG & \multirow{2}{*}{$\begin{array}{l}\text { For amplification of the } 2137 \mathrm{bp} \text { of the } h p h \\
\text { cassette from plasmid pSKH }\end{array}$} \\
\hline hph R & CTAGAAAGAAGGATTACCTC & \\
\hline pigG d3F & GAGGTAATCCTTCTTTCTAGGTGCCGATCAAGACGAAGGA & \multirow{2}{*}{$\begin{array}{l}\text { For amplification of the } 867 \mathrm{bp} \text { of } 3^{\prime} \text { flanking } \\
\text { regions of the mrpig } G \text { gene }\end{array}$} \\
\hline pigG d3R & CTCTTCCAGCAGGACCAACT & \\
\hline pigG c5F & CCAGACACCGAACAGCCGCA & \multirow{2}{*}{$\begin{array}{l}\text { For amplification of the } 1823 \mathrm{bp} \text { of } 5^{\prime} \text { flanking } \\
\text { regions and open reading frame (ORF) of the } \\
\text { mrpigG gene }\end{array}$} \\
\hline pigG c5R & GGTTACGGTTCGATGGGGTTGAGTTGGGTCTCGCTGTAGGGCTGGAT & \\
\hline G418 F & CCAACTCAACCCCATCGAACCGTAACC & \multirow{2}{*}{$\begin{array}{l}\text { For amplification of the } 1221 \mathrm{bp} \text { of the neo } \\
\text { cassette from plasmid pKN1 }\end{array}$} \\
\hline G418 R & ATCATCATGCAACATGCATG & \\
\hline pigG c3F & CATGCATGTTGCATGATGATCGATCTTCTTCGCAGACACC & \multirow{2}{*}{$\begin{array}{l}\text { For amplification of the } 860 \text { bp of } 3^{\prime} \text { flanking } \\
\text { regions of the mrpig G gene }\end{array}$} \\
\hline pigG c3R & CGTCACTCGCTTCCAGGTCG & \\
\hline pigG ov5F & CAGACATACTGCTAAACTCG & \multirow{2}{*}{$\begin{array}{l}\text { For amplification of the } 969 \mathrm{bp} \text { of } 5^{\prime} \text { flanking } \\
\text { regions of the mrpig } G \text { gene }\end{array}$} \\
\hline pigG ov5R & GGTTACGGTTCGATGGGGTTGAGTTGGGGTGCGGTGCTGGCGAGAGT & \\
\hline ptrpC F & CATGCATGTTGCATGATGATGTCGACAGAAGATGATATTG & \multirow{2}{*}{$\begin{array}{l}\text { For amplification of the } 373 \mathrm{bp} \text { of the } p t r p C \\
\text { promoter from plasmid pKSH }\end{array}$} \\
\hline ptrpC R & CATATCGATGCTTGGGTAGA & \\
\hline mrpigG ov3F & TATTCTACCCAAGCATCGATATG ATGCCAGCCAACCGCTCCAG & \multirow{2}{*}{$\begin{array}{l}\text { For amplification of the } 1621 \text { bp of } 3^{\prime} \text { flanking } \\
\text { regions and ORF of the mrpigG gene }\end{array}$} \\
\hline pigG ov3R & TCTTCCAGCAGGACCAACTC & \\
\hline pigG F2 & GCGCTGGCTGCGCTCAT & \multirow{2}{*}{$\begin{array}{l}\text { For amplification of the } 503 \mathrm{bp} \text { of the partial } \\
\text { mrpigG gene }\end{array}$} \\
\hline pigG R2 & ССТСССАСТССАТААССС & \\
\hline pigG qF & GGGTGAATGGGCGGGACTA & \multirow{2}{*}{ For real-time qPCR analysis of mrpigG(144bp) } \\
\hline pigG qR & GCCAGCAATACGGCAAAGC & \\
\hline GAPDH F & CAAGCTCACTGGCATGTCTATG & \multirow{2}{*}{ For real-time qPCR analysis of GAPDH (243bp) } \\
\hline GAPDH R & AAGTTCGAGTTGAGGGCGATA & \\
\hline
\end{tabular}

\subsection{Deletion, Complementation and Overexpression of the mrpigG Gene}

To verify the function of mrpigG, the gene was deleted, complemented and overexpressed, according to the homologous recombination strategy, as previously described $[14,15]$. The mrpigG gene deletion cassette (5'UTR-hph-3'UTR), complementation cassette (5'UTR-mrpigG-neo-3'UTR) and overexpression cassette (5'UTR-neo-PtrpC-mrpigG-3'UTR) were constructed by double-joint PCR, and are shown schematically in Figures 1a, 2a and 3a, respectively. The relative primer pairs are shown in Table 2.

The gene disruption construct carried $5^{\prime}$ flanking regions (884 bp, amplified with the primer pair pigG d5F and pigG d5R), hygromycin B resistance gene ( $h p h, 2137 \mathrm{bp}$, amplified from plasmid pSKH) with the primer pair hph $\mathrm{F}$ and hph $\mathrm{R}$ ), and 3' flanking regions (867 bp, amplified using the primer pair pigG d3F and pigG d3R), consecutively.

The gene complementation structure possessed $5^{\prime}$ flanking, together with mrpigG open reading frame (ORF) regions (1823 bp, amplified with the primer pair pigG c5F and pigG c5R), neomycin phosphotransferase resistance gene $(n e 0,1221 \mathrm{bp}$, amplified from plasmid pKN1 with the primer pair G418 F and G418 R) and 3' flanking regions (860 bp, amplified with the primer pair pigG c3F and pigG c3R), consecutively.

The gene overexpression construct carried $5^{\prime}$ flanking regions (969 bp, amplified with the primer pair pigG ov5F and pigG ov5R), neomycin phosphotransferase resistance gene (neo, $1221 \mathrm{bp}$, amplified from plasmid pKN1 with the primer pair G418 F and G418 R), the trpC promoter (373 bp, amplified 
from plasmid pSKH using the primer pair PtrpC F and PtrpC R) and the mrpigG gene together with $3^{\prime}$ flanking regions (1621 bp, amplified with the primer pair pigG ov3F and pigG ov3R), consecutively.

Three abovementioned structures were separately cloned into pMD19-T (Takara, Dalian, China), then all the cloned fusion DNA fragments and plasmid pCAMBIA3300 were digested with KpnI and HindIII, and were ligated by T4 DNA ligase to generate plasmids pCPIGG, pCCPIGG and pCOPIGG, respectively. Then three plasmids were separately transformed into Agrobacterium tumefaciens EHA105 using a freeze-thaw method. Then, three $A$. tumefaciens EHA105 clones containing pCPIGG, pCOPIGG were incubated for transformation with $M$. ruber $\mathrm{M} 7$ to yield the mrpigG disruption strains ( $\Delta$ mrpigG), mrpigG and overexpression strains (M7::PtrpC-mrpigG), respectively. Meanwhile, the A. tumefaciens EHA105 clones containing pCCPIGG were incubated for transformation with the $\triangle m r p i g G$ strain to generate the mrpigG complementation strains ( $\Delta$ mrpigG::mrpigG).

\subsection{Real-Time $q P C R$ Analysis}

The expression of mrpigG in the selected $\Delta$ mrpigG, $\Delta$ mrpigG::mrpigG, M7::PtrpC-mrpigG strain and the wild-type strain (M. ruber M7) was analyzed by real-time quantitative PCR analysis (RT-qPCR). One milliliter freshly harvested spores $\left(10^{5} \mathrm{cfu} / \mathrm{mL}\right)$ of each strain were inoculated into PDA medium and incubated at $28^{\circ} \mathrm{C}$, and samples were taken every other day from the 3rd day to the 11th day. RT-qPCR was performed according to the method described by Liu et al. [14]. GAPDH was used as a reference gene. The primers used in these analyses are listed in Table 2.

\subsection{MP and Citrinin Analyses}

Previous studies have revealed that MPs accumulate mainly in the mycelia, where citrinin exists in media [16]. Thus, intracellular MPs and the extracellular citrinin were detected. One milliliter of freshly harvested spores $\left(10^{5} \mathrm{cfu} / \mathrm{mL}\right)$ of each strain were inoculated on PDA plates covered with cellophane membranes, and incubated at $28^{\circ} \mathrm{C}$ for 11 days. The samples were taken every 2 days from the 3rd day to the 11th day of culture to measure the MPs and CIT production [5]. Twenty milligrams of freeze-dried mycelia or media powder were suspended in $1 \mathrm{~mL} 80 \%(v / v)$ methanol solution, and subjected to 30 min ultrasonication treatment (KQ-250B, Kunshan, China).

Then, MPs were separated by an ACQUITY UPLC BEH C18 column $(2.1 \mathrm{~mm} \times 100 \mathrm{~mm}, 1.7 \mu \mathrm{m}$, Waters), and detected on a Waters ACQUITY UPLC I-class system by UV-Vis spectra (Waters, Milford, MA, USA). A gradient elution was performed with the mobile phase including solvent A (acetonitrile), solvent B (ultra-pure water) and solvent C ( $0.1 \%$ formic acid in water) with a flow rate of $0.3 \mathrm{~mL} / \mathrm{min}$ and an injection volume of $2 \mu \mathrm{L}$. The gradient elution was performed as follows: firstly, Solvent $\mathrm{A} / \mathrm{B} / \mathrm{C}$ maintained 35:55:10 for $3 \mathrm{~min}$, then the content was changed to 75:15:10 for $15 \mathrm{~min}$, followed by adjusting to 90:0:10 for $5 \mathrm{~min}$. Subsequently, the solvent was changed to 35:55:10 for $5 \mathrm{~min}$. Finally, the column was equilibrated with 35:55:10 for $3 \mathrm{~min}$.

The citrinin was detected on a Waters ACQUITY UPLC system by means of a fluorescence detector (Waters, Milford, MA, USA) A gradient elution was performed with the mobile phase including solvent A ( $0.1 \%$ formic acid in water) and solvent $B$ (acetonitrile) with a flow rate of $0.3 \mathrm{~mL} / \mathrm{min}$ and an injection volume of $2 \mu \mathrm{L}$. The gradient elution was performed as follows: Solvent $\mathrm{A} / \mathrm{B}$ was first maintained at 90:10 for $3 \mathrm{~min}$. Secondly, the content of solvent A was decreased from $90 \%$ to $30 \%$ for $7 \mathrm{~min}$. Then, the amount of solvent A was decreased from $30 \%$ to $10 \%$ for $0.01 \mathrm{~min}$ and was maintained for $3 \mathrm{~min}$. Finally, the amount of solvent A was increased from $10 \%$ to $90 \%$ for $3 \mathrm{~min}$. The temperature of the chromatographic column and samples were maintained at $40{ }^{\circ} \mathrm{C}$ and $4{ }^{\circ} \mathrm{C}$, respectively.

\section{Results}

\subsection{Sequence Analysis of the mrpigG Gene in M. ruber M7}

A 1.24-kb fragment containing the putative mrpig $G$ homolog was successfully amplified from the genomic DNA of M. ruber M7. Sequence prediction of mrpigG by SoftBerry's FGENESH program 
revealed that the putative $m$ rpigG gene consists only of an 822 bp open reading frame (ORF) which consists of 1 exon and encodes 273 amino acids. A database search with the Pfam 33.1 program showed that MrPigG pertains to the serine hydrolase family (FSH). A database search with NCBI-BLAST demonstrated that the deduced 273 -amino acid sequence encoded by $m r p i g G$ shares $93.01 \%$ similarity with the amino oxidase/esterase of M. pilosus (GenBank: AGN71609.1), 69.26\% similarity with serine hydrolase FSH of Penicillium occitanis (GenBank: PCH03976.1), and 55.72\% similarity with the serine hydrolase-domain-containing protein of Pseudomassariella vexata (GenBank: ORY68205.1).

\subsection{Verification of the mrpigG Deletion, Complementation and Overexpression Strains}

After the plasmid pCPIGG was transformed into M. ruber M7, transformants with hygromycin B resistance were obtained and verified by PCR, and three of 115 were transformant mrpigG-deleted strains. As shown in Figure 1c, no DNA band was amplified when genomic DNA of one mrpigG disruptant named $\Delta m r p i g G$ was used as template with primers pigG F2-pigG R2, although a 0.5-kb product appeared using the genome of the wild-type strain M. ruber M7. A 2.14-kb fragment of the $h p h$ gene could be amplified from $\triangle m r p i g G$ using primers hph F-hph $\mathrm{R}$, while nothing was obtained from M. ruber M7. Meanwhile, amplicons of M. ruber M7 (2.28 kb) and $\Delta m r p i g G ~(3.89 \mathrm{~kb})$ differed in size when primers pigG d5F-pigG d3R annealing to homologous arms (5' flanking and $3^{\prime}$ flanking regions) were used.

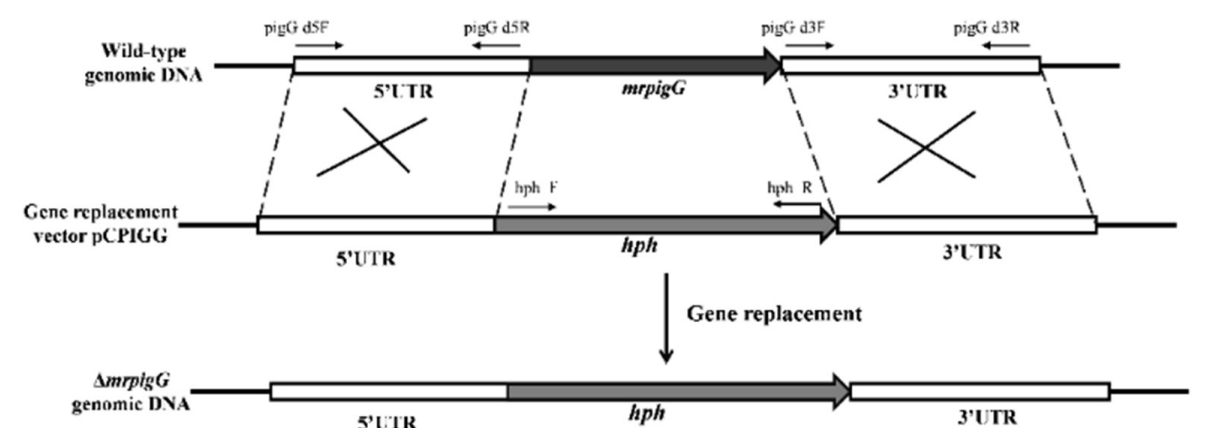

(a)

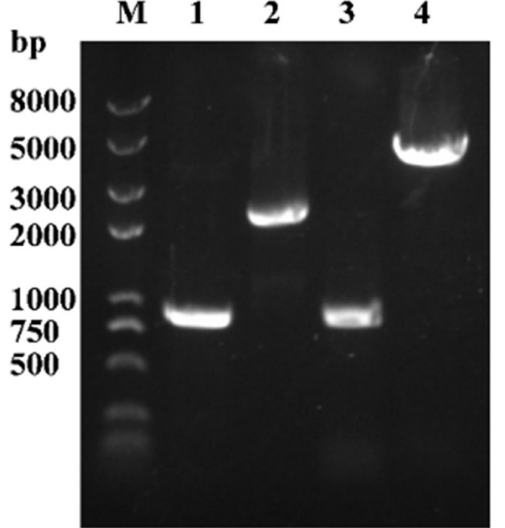

(b)

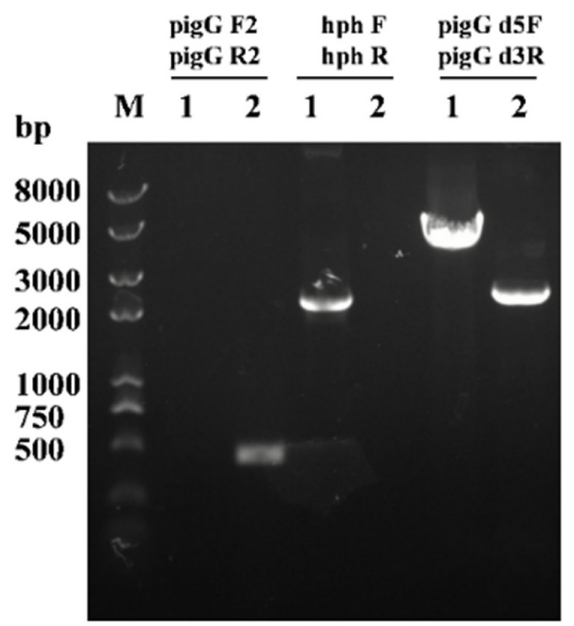

(c)

Figure 1. Deletion of mrpigG in M. ruber M7. (a) Schematic representation of the homologous recombination strategy yielding mrpigG deletion strains. (b) Construction of mrpig $G$ disruption construct by double-joint PCR. Lane 1, 5' flanking region of mrpig G; lane 2, hygromycin resistance cassette; lane 3, 3' flanking region of mrpigG; lane 4, double-joint PCR product. (c) Confirmation of mrpigG homologous recombination events. Three primer pairs were used, and PCR amplifications showed distinct bands in different strains. Lane 1, the $\Delta$ mrpigG strain; lane 2, the wild-type strain. 
Three $\Delta$ mrpigG::mrpigG strains with G418 resistance were obtained. PCR analysis of one of the $\Delta$ mrpigG::mrpigG strains is shown in Figure 2c. A 0.5-kb product was amplified when the genomic DNA of $\Delta$ mrpigG::mrpigG was used as template with primers pigG F2-pigG R2, whereas no DNA band was amplified using the genome of $\Delta m r p i g G$. A 1.22-kb fragment of the neo gene could be amplified from $\Delta$ mrpigG::mrpigG using primers G418 F-G418 R, whereas nothing was obtained from $\Delta m r p i g G$. A 2.14-kb product of the $h p h$ gene could be amplified from $\Delta m r p i g G$ using primers hphF-hphR, whereas nothing was obtained from $\Delta$ mrpigG::mrpigG. The neo fragment replaced the $h p h$ gene, which might reveal that it was a successful homologous recombination event. Meanwhile, amplicons of $\Delta$ mrpigG::mrpigG $(3.9 \mathrm{~kb})$ and $\Delta$ mrpigG $(4.3 \mathrm{~kb})$ differed in size when primers pigG d5F-pigG $\mathrm{d} 3 \mathrm{R}$, annealing to homologous arms, were used.

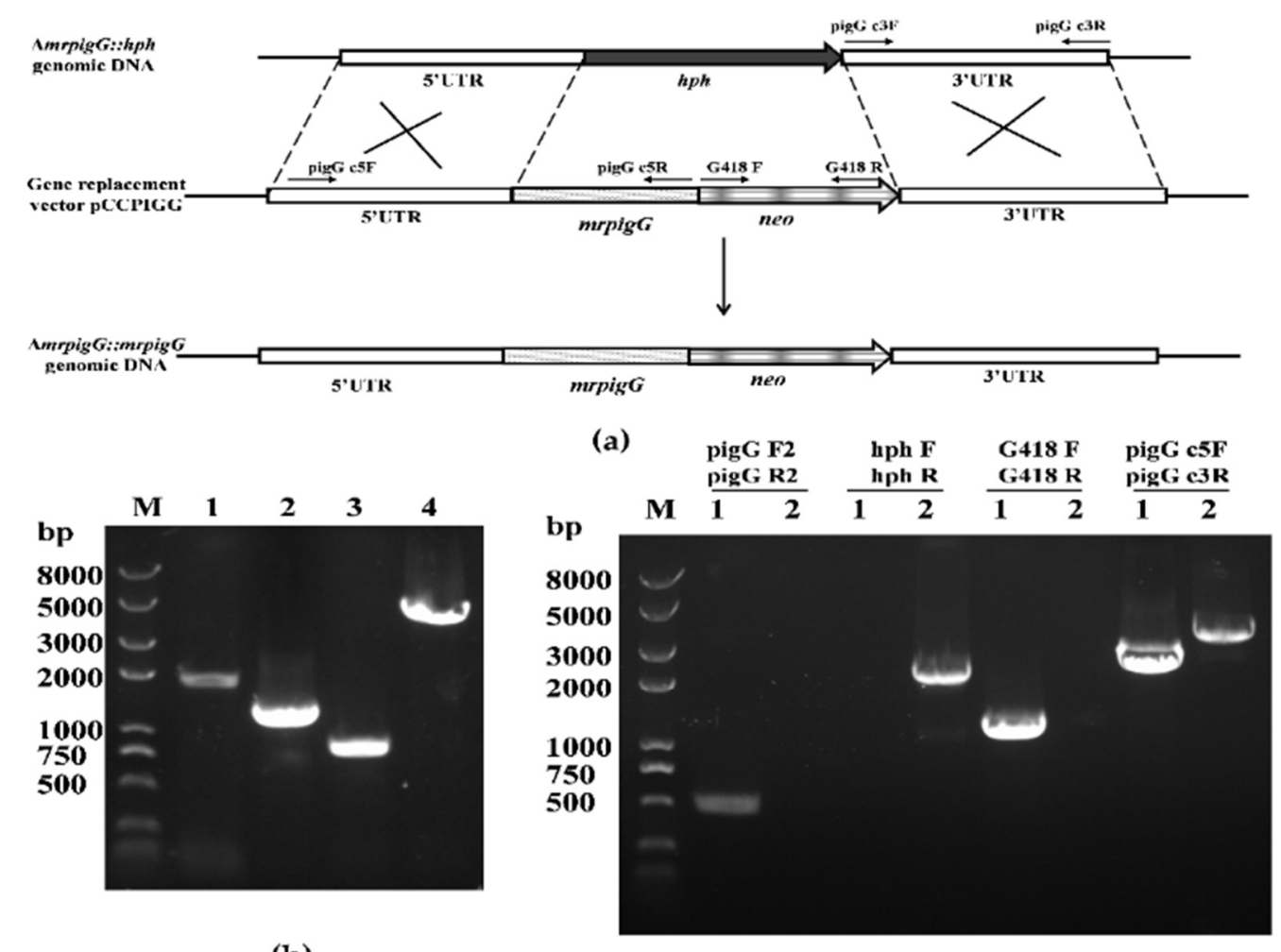

(b)

(c)

Figure 2. Complementation of mrpigG in M. ruber M7. (a) Schematic representation of the homologous recombination strategy to construct mrpigG complementation strains (b) Construction of mrpigG complementation cassette by double-joint PCR. Lane 1, 5' flanking region of mrpigG plus mrpigG ORF regions; lane 2, G418 resistance cassette; lane 3, 3' flanking region of mrpigG; lane 4, double-joint PCR product. (c) Confirmation of mrpigG homologous recombination events. Four primer pairs were used, and PCR amplifications showed distinct bands in different strains. Lane 1, the $\Delta$ mrpigG:: mrpigG strain; lane 2, the $\Delta$ mrpigG strain.

Seven M7::PtrpC-mrpigG strains with G418 resistance were obtained and checked by PCR analysis. As shown in Figure 3c, amplicons of M. ruber M7 and M7::PtrpC-mrpigG were equal in size when primers pigG F2-pigG R2 were used, and the size was $0.5 \mathrm{~kb}$. A $1.22-\mathrm{kb}$ fragment of the neo gene and a $0.37-\mathrm{kb}$ product of the PtrpC gene could be amplified from M7::PtrpC-mrpigG using primers G418 F-G418 R and PtrpC F-PtrpR, whereas nothing was obtained from M7, respectively. Meanwhile, amplicons of M7::PtrpC-mrpigG (4.13 kb) and M. ruber M7 (2.56 kb) differed in size when primers pigG d5F-pigG d3R annealing to homologous arms were used, which proved that there was a single copy of the PtrpC-mrpigG overexpression construct integrated in M7::PtrpC-mrpigG. 


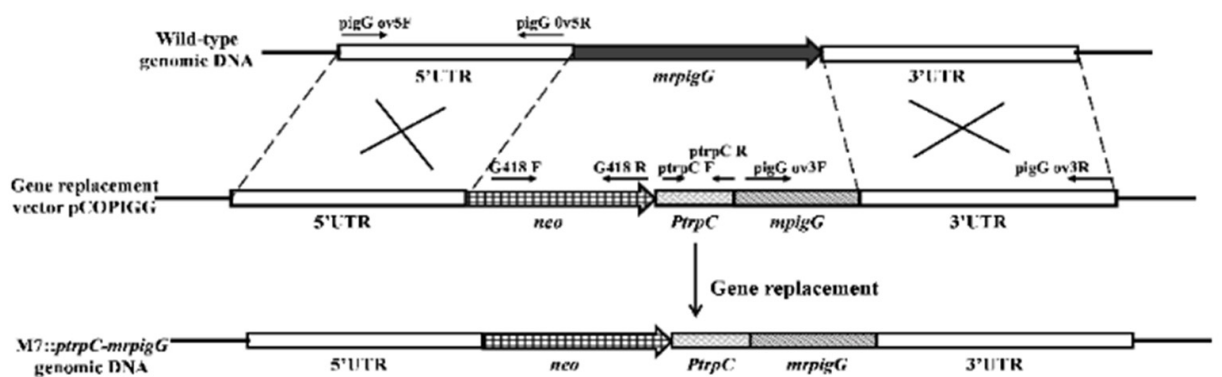

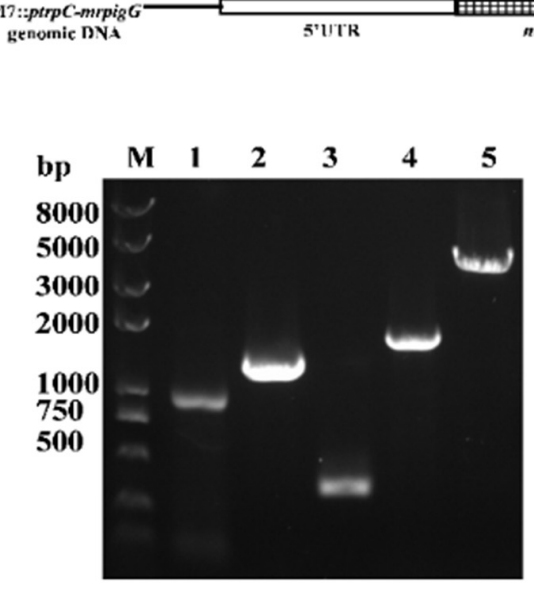

(b) (a)

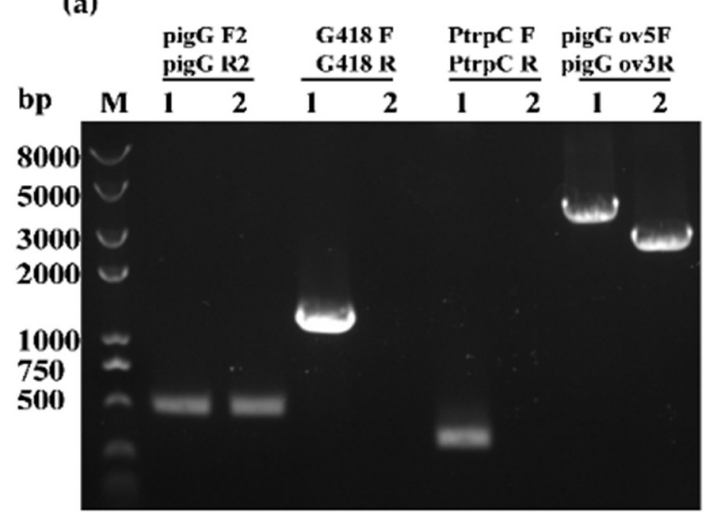

(c)

Figure 3. Overexpression of mrpigG in M. ruber M7. (a) Schematic representation of the homologous recombination strategy yielding mrpigG overexpression strains. (b) Construction of mrpigG overexpression construct by double-joint PCR. Lane 1, 5' flanking region of mrpigG; lane 2, G418 resistance cassette; lane 3, the $\operatorname{trpC}$ promoter; lane 4, mrpigG ORF regions plus 3' flanking region of mrpigG; lane 5, double-joint PCR product. (c) Confirmation of mrpigG homologous recombination events. Three primer pairs were used, and PCR amplifications showed distinct bands in different strains. Lane 1, the M7::PtrpC-mrpigG strain; lane 2, the wild-type strain.

\subsection{RT-qPCR Analysis of $\triangle$ mrpigG, $\triangle$ mrpigG::mrpigG, M7::PtrpC-mrpigG and M. ruber M7}

The transcription levels of the $\Delta$ mrpigG gene in the four strains- $\Delta$ mrpigG, $\Delta$ mrpigG::mrpigG, M7::Ptrp C- $\Delta$ mrpigG and the wild-type strain M. ruber M7-were analyzed by RT-qPCR. As shown in Figure 4, $\Delta$ mrpigG was deficient in the expression of the mrpigG gene, which further verified the success of the gene knockout. The tendency of mrpigG expression in $\Delta$ mrpigG::mrpigG was similar with that of M. ruber M7, and the average level of mrpigG expression in M7::PtrpC-mrpigG was higher than that of M. ruber M7, in spite of the 3rd day.

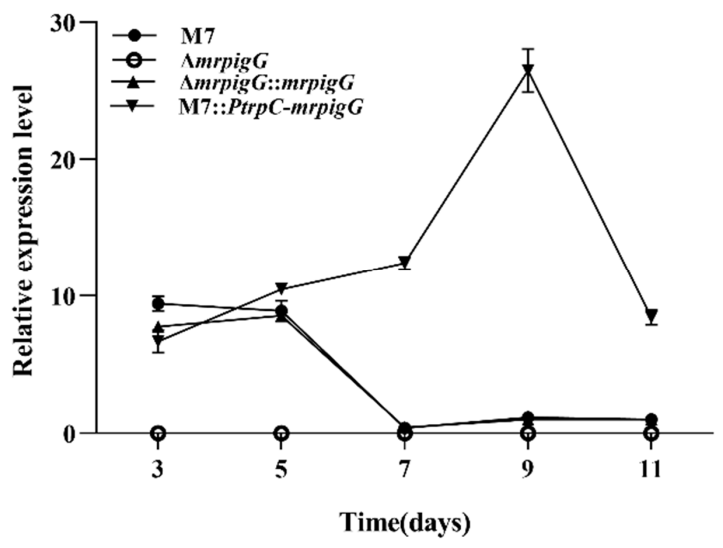

Figure 4. RT-qPCR analysis of the mrpigG gene in the mrpigG deletion strain, the mrpigG complementation strain, the mrpigG overexpression strain and the wild-type strain. 


\subsection{Morphologies and Biomasses of $\Delta m r p i g G, \Delta m r p i g G::$ mrpigG, M7::PtrpC-mrpigG and M. ruber M7}

Colonial and microscopic characteristics of M. ruber M7 were observed on the different media (PDA, CYA, MA, G25N) to investigate the influence of the mrpigG deletion, complementation and overexpression on developmental processes. The results showed that colonial morphologies (Figure 5a) and microscopic morphologies, including conidia and cleistothecia, of $\Delta m r p i g G, \Delta m r p i g G:: m r p i g G$ and M7::PtrpC-mrpigG were not significantly different from those of $M$. ruber M7 on different culture plates (Figure 5b). Moreover, those four strains showed similar biomasses on PDA (Figure 5c).

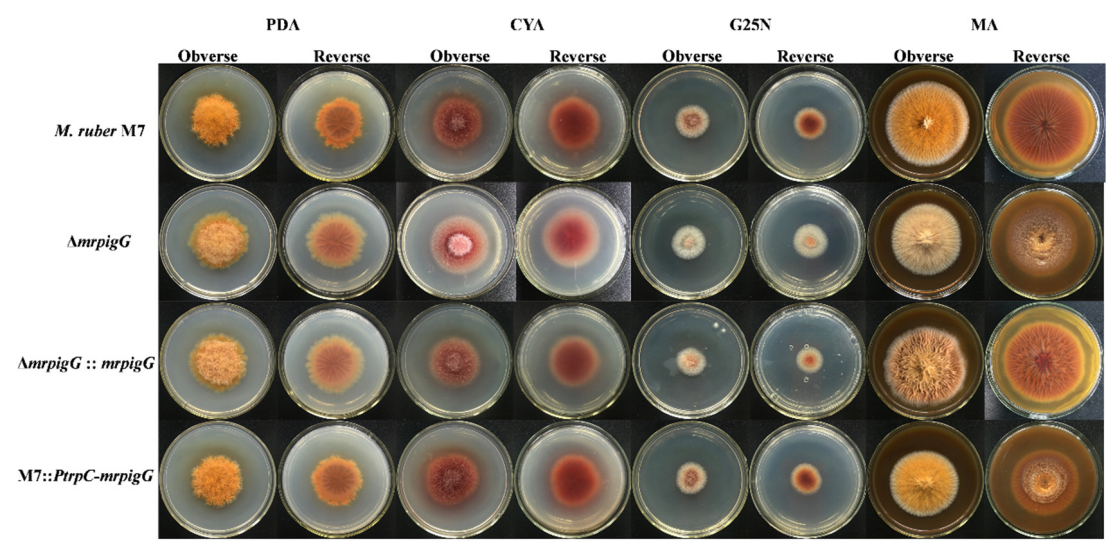

(a)

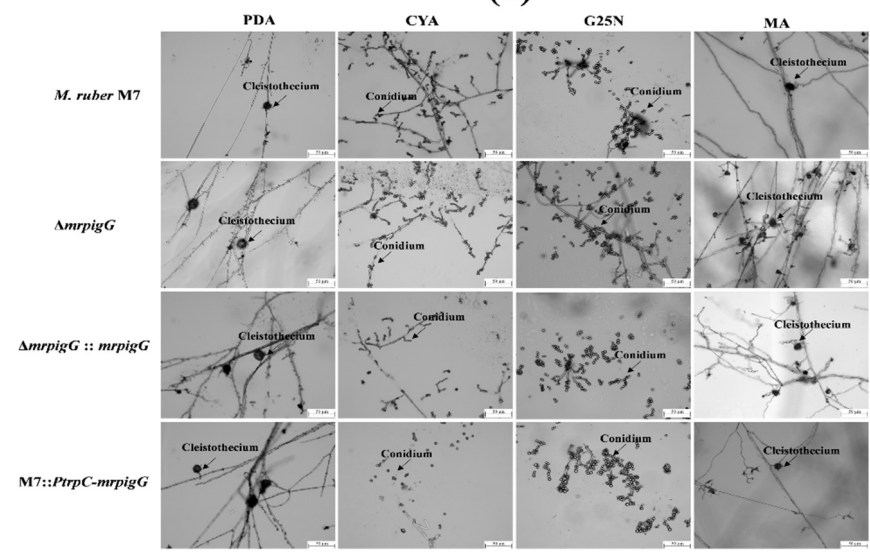

(b)

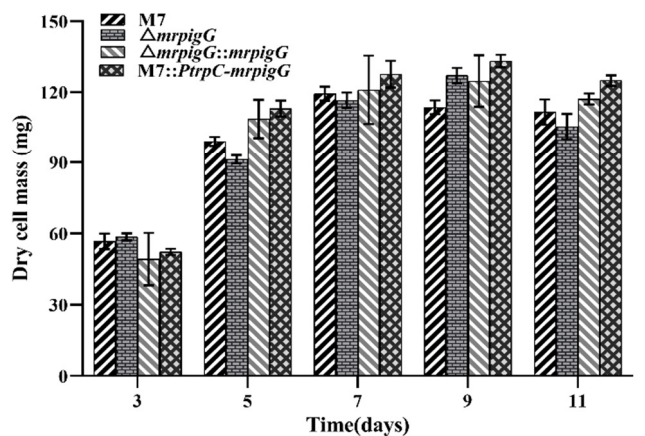

(c)

Figure 5. Morphologies and biomasses of $\Delta$ mrpigG, $\Delta$ mrpigG::mrpigG, M7::PtrpC-mrpigG and M. ruber M7. (a) Colonial morphologies on potato dextrose agar (PDA), Czapek yeast extract agar (CYA), malt extract agar (MA) and glycerol nitrate agar (G25N) plates. (b) Cleistothecia and conidia formation (at 10 days) of the M. ruber M7 and the mrpigG transformants on different plates (PDA, CYA, G25N and MA) at $28^{\circ} \mathrm{C}$. (c) Biomass (dry cell weight). Experiments were performed in triplicate. Error bars indicate standard deviation. 


\subsection{MP and CIT Production Analysis of $\triangle m r p i g G, \Delta m r p i g G:: m r p i g G, M 7:: P t r p C-m r p i g G$ and M. ruber M7}

Previous studies [5,9] have demonstrated that $M$. ruber M7 can produce MPs and CIT, but no MK, so the yields of the eight main MPs (four yellow pigments: monasfloure A, monascine, monasfloure B and ankaflavin; two orange pigments: rubropunctatin and monascorubrin; and two red pigments: rubropunctamine and monascorubramine) (Figure 6) and CIT in M. ruber M7 and its mutants were analyzed in this study to uncover the effect of mrpigG on SMs. As for the yellow pigment production, the levels of monasfloure A, monascine, monasfluore B and ankaflavin in $\triangle$ mrpigG were $30.7 \%, 40.1 \%$, $67.2 \%$ and $94 \%$ of that in M. ruber M7, respectively (Figure 7a-d). For orange and red pigment production, the production of rubropunctatin (an orange pigment (OP)) and rubropunctamine (a red pigment (RP)) in $\Delta$ mrpigG, both of which contain a 5-carbon side chain, were $57.7 \%$ and $22.3 \%$ of those in M. ruber M7 (Figure 7e-f). On the other hand, monascorubrin (an OP) and monascorubramine (an RP) in $\Delta$ mrpigG, both of which have a 7-carbon side chain, were 1.15 and 2.55 times those of $M$. ruber M7 (Figure $7 \mathrm{~g}-\mathrm{h}$ ).

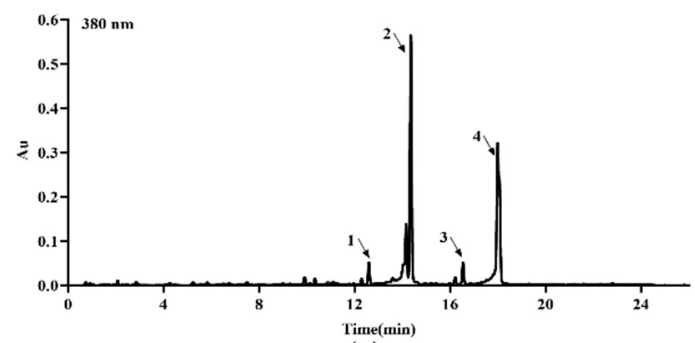

(a)

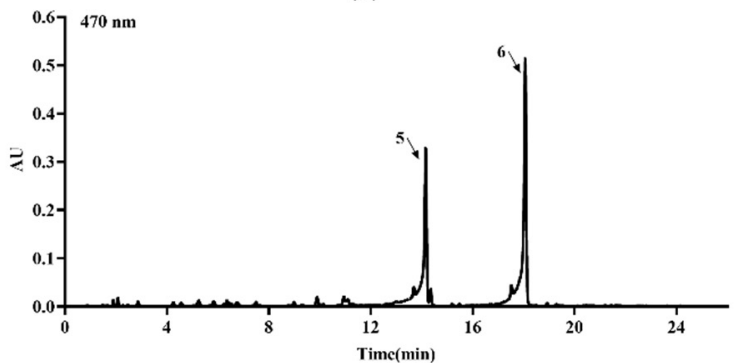

(b)

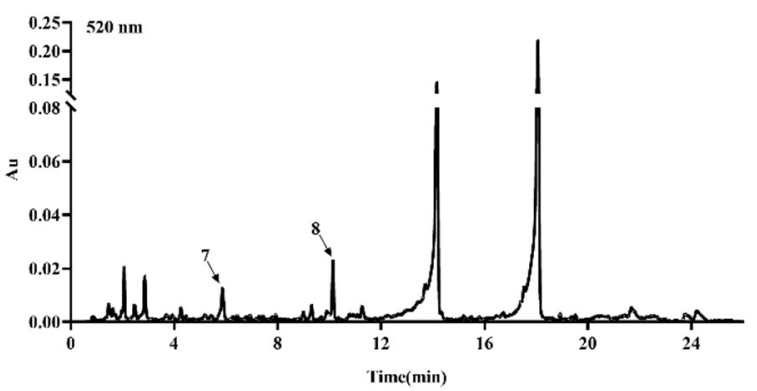

(c)

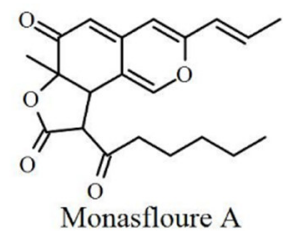

(1)

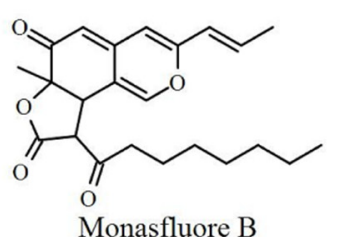

(3)<smiles>C/C=C/C1=CC2=CC3=C(C(=O)CCCCC)C(=O)OC3(C)C(=O)C2=CO1</smiles>

Rubropunctatin

(5)<smiles></smiles>

Rubropunctamine (7)<smiles>C/C=C/C1=CC2=C(CO1)C(=O)C1(C)OC(=O)C(C(=O)CCCCC)C1C2</smiles>

(2)<smiles>C/C=C/C1=CC2=C(CO1)C(=O)C1(C)OC(=O)C(C(=O)CCCCCC)C1C2</smiles>

(4)<smiles>C/C=C/C1=CC2=CC3=C(C(=O)C(=O)CCCCCC)C(=O)C(C)(OC3=O)C2=CO1</smiles>

(6)<smiles></smiles>

Monascorubramine

(8) (d)

Figure 6. The main pigments of M. ruber M7 detected by UPLC. (a) The chromatogram of 4 main yellow pigments at $380 \mathrm{~nm}$, which are indicated by 1, 2, 3 and 4. (b) The chromatogram of 2 main orange pigments at $470 \mathrm{~nm}$, which are indicated by 5 and 6 . (c) The chromatogram of the 2 main red pigments at $520 \mathrm{~nm}$, which are indicated by 7 and 8 . (d) The chemical structure formula of the 8 pigments. 

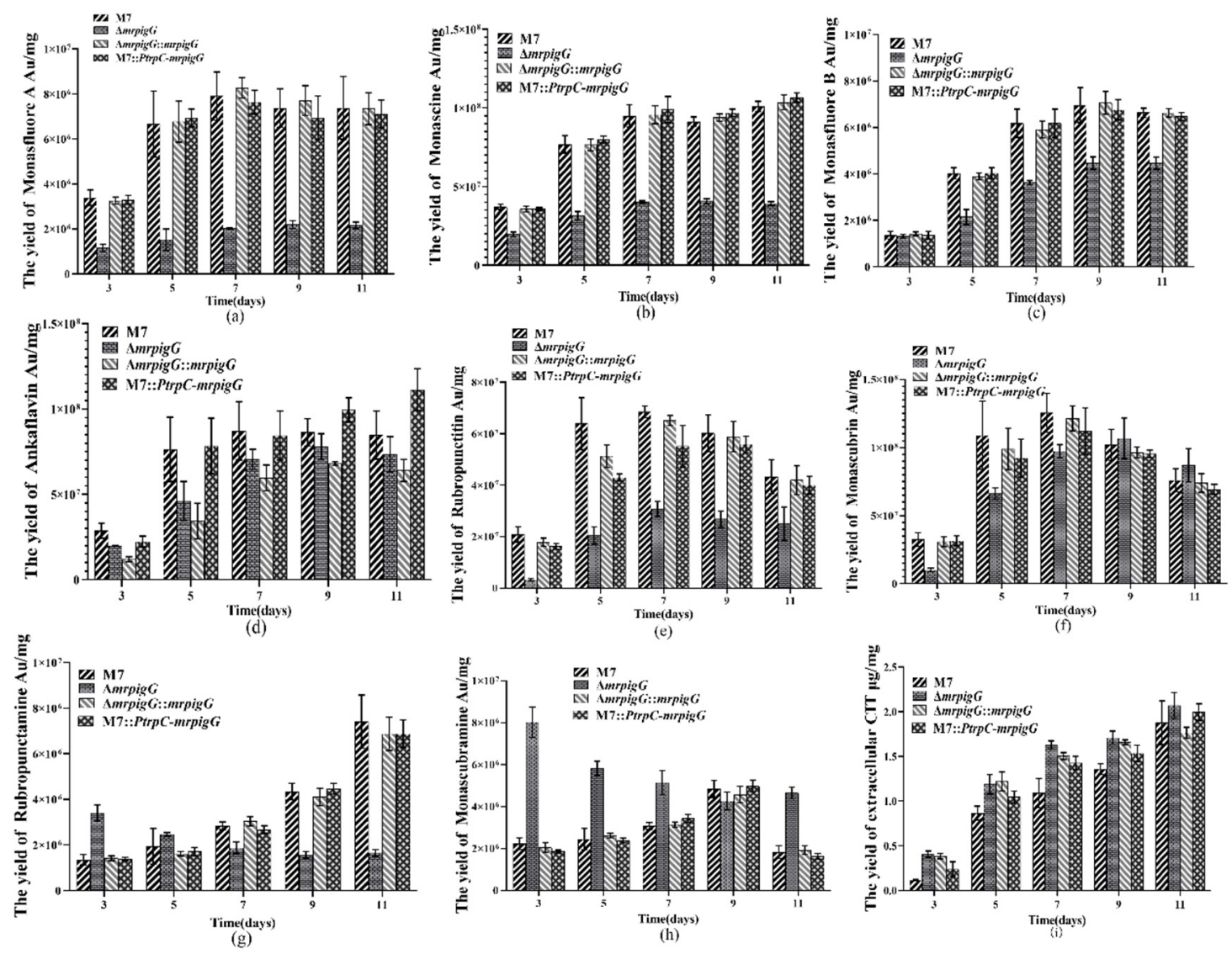

Figure 7. Production of pigments and citrinin by the wild-type strain M. ruber M7 and the mrpigG transformants. (a) The yield of monasfloure A. (b) The yield of monascine. (c) The yield of monasflore B. (d) The yield of ankaflavin. (e) The yield of intracellular rubropunctatin. (f) The yield of monascorubrin. (g) The yield of rubropunctamine. (h) The yield of monascorubramine. (i) The yield of extracellular citrinin (CIT). The error bar represents the standard deviation between the three repeats. Capitals signify $p$-value $<0.01$

As shown in Figure 7i, the tendency of all strains' extracellular citrinin production was always increased from the 3rd day to the 11th day of cultivation in PDA media. During the early stage (3-7 days) the CIT production in M. ruber M7 was only $22.8-72.9 \%$ that of $\Delta m r p i g G$, whereas the other two mutants possessed similar yields to those of $\Delta$ mrpigG. At the end of the 11 days of fermentation, extracellular citrinin concentration of the four strains were similar to those of the wild-type strain $M$. ruber $\mathrm{M} 7$, at $1.88 \pm 0.25 \mu \mathrm{g} / \mathrm{mg}$ citrinin.

\section{Discussion}

MPs with a common azaphilone skeleton are a large group of secondary metabolites produced via the polyketide pathway by filamentous fungi, mainly by Monascus spp.and Penicillium spp. [1,17]. MPs have been used in the food coloring, pharmaceutical, cosmetics, textile, printing and dyeing industries [3,18]. Recently, researchers proposed a MP biosynthetic pathway, in order to explore the generation of the bewildering diversity and complexity of MPs. To date, the functions of many genes in the MP gene clusters have been well investigated in M. ruber M7 and M. purpureus KACC42430, but there has been limited research on a few related genes, such as mpigG, mpigH and mpigI [8].

In current study, the generation of mrpig $G$ mutants by homologous recombination, as shown in Figures 5 and 7, demonstrated that mrpig $G$ was a member of the MP gene cluster. Searched with the Pfam 33.1 program, the data showed that $m r p i g G$ belonged to the serine hydrolase family, which is a large, ubiquitous family of enzymes grouped based on their ability to perform hydrolysis reactions on 
a range of biological substrates, such as ester, thioester, amide and epoxide bonds in small molecules, peptides or proteins [19-21]. The majority of serine hydrolases ( $>60 \%$ ) adopt an $\alpha, \beta$-hydrolase fold and employ a Ser-His-Asp catalytic triad [21], but more than half of the serine hydrolases ( $>120$ enzymes) remain poorly annotated, with no described physiological function or identified substrates $[19,22]$.

In this study, the results showed that there was a 10-70\% decrease in the four typical yellow pigments by mrpigG deletion. Simultaneously, the yields of typical orange pigments (OPs) and red pigments (RPs) revealed a completely different accumulation. In detail, compared with M. ruber M7, the yields of rubropunctatin (an OP) and its derivative rubropunctamine (an RP) produced by $\Delta m r p i g G$ reduced to $57.7 \%$ and $22.3 \%$, respectively. On the contrary, the production of monascorubrin (an OP) and its derivative monascorubramine (an RP) increase by 0.15 -fold and 1.55-fold, respectively. These two OPs and RPs separately possessed the same azaphilone skeleton. In the same vein, rubropunctatin $(\mathrm{OP})$ and rubropunctamine (RP) contained the $-\mathrm{C}_{5} \mathrm{H}_{11}$ side chain, wheres the monascorubrin (OP) and monascorubramine (RP) owned the $-\mathrm{C}_{7} \mathrm{H}_{15}$ side chain. The serine hydrolase encoded by mpig $G$ specifically catalyzed the synthetic compounds with the $-\mathrm{C}_{5} \mathrm{H}_{11}$ side chain, and might show substrate specificity to a certain extent. Navia-Paldanius et al. delineated the substrate preferences of three serine hydrolases by analyzing accumulation of fatty acid esters in cell lysates individually overexpressing each of the enzymes in assay mixes containing different chain length and saturation acylglycerols [23]. Ritchie et al. elaborated that the human type II thioesterase TE2 (containing a Ser-Asp-His catalytic triad) preferred an engineered human acyl-ACP substrate releasing short chain fatty acids from full-length fatty acid synthase (FASN) during turnover. The ability of TE2 to release fatty acids from FASN was involved in polyketide and non-ribosomal peptide synthase synthases [24].

Taking OP and RP production into account, the function of mrpigG in the biosynthesis pathway of MPs was proposed, as shown in Figure 8. In the proposed pathway, the $\beta$-ketooctanoic or $\beta$-ketodecanoic acid moieties used for this acylation step (P2) are produced by a dedicated two-subunit fatty acid synthetase (MrPigJ and MrPigK in M. ruber M7) encoded in the MP gene clusters. The $\beta$-keto fatty acid, along with ACP, specially hydrolyzes by means of MrPigG, then the hydrolysates are transferred to the acyltransferase (MrPigD). The C-4 alcohol of the benzopyran in P1 is then acylated with a medium chain $\beta$-keto fatty acid by MrPigD to yield the putative intermediate $\mathrm{P} 2$, which has been characterized as a precursor of MPs $[8,9,25]$. MrPigG may act as a metabolic serine hydrolase, like type II TE proteins, promoting the release of the short chain compounds from the thioester moiety on ACP $[10,26]$.

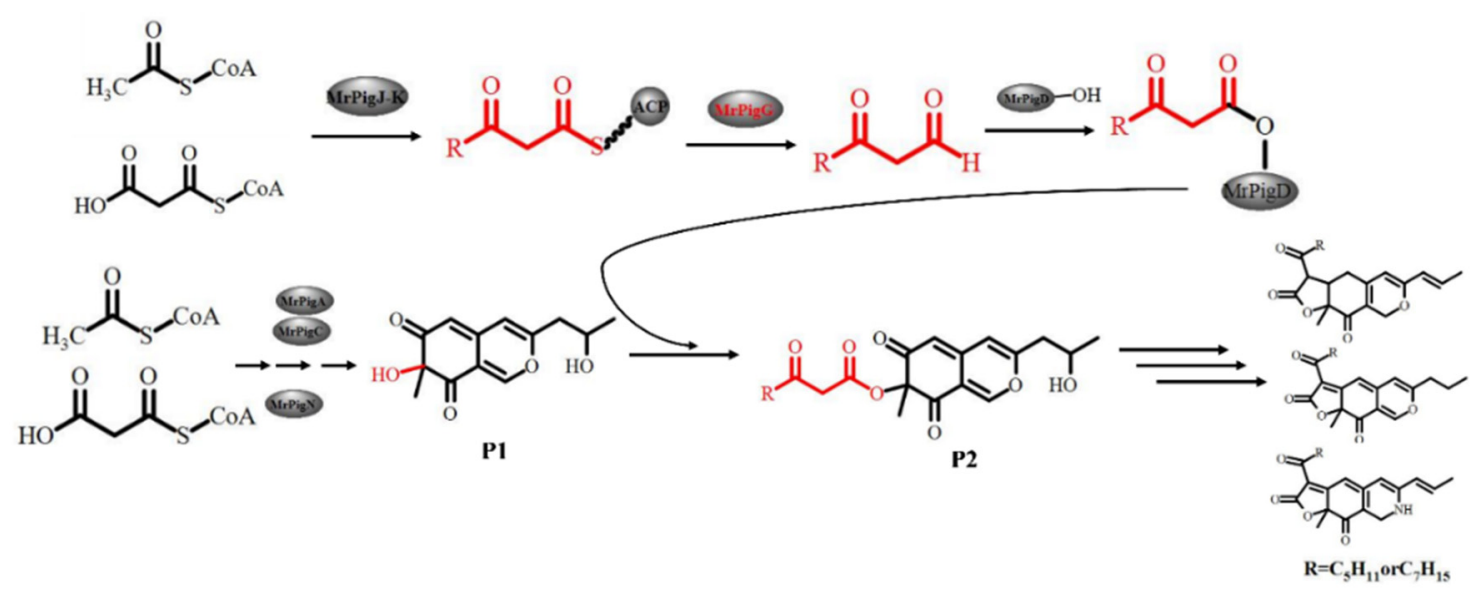

Figure 8. Proposed pathway involving the MrPigG protein.

In conclusion, the mrpigG gene pertains to the MP biosynthetic gene cluster of M. ruber and plays an unignorable role in the biosynthesis of MPs. The disruption, complementation and overexpression of mrpig $G$ had very little effect on the biomass, citrinin production and morphology of the strains. Compared with M. ruber M7, $\Delta$ mrpigG equally yielded eight typical pigments, but the production of 
four yellow pigments was partially restrained, and the synthesis of orange and red pigments depended on side chain specificity. The content of rubropunctatin (OP) and its derivative rubropunctamine (RP), both of which have a five-carbon side chain, were $57.7 \%$ and $22.3 \%$ of those in M. ruber M7. On the other hand, monascorubrin (OP) and its derivative monascorubramine (RP), both of which have a seven-carbon side chain, were 1.15 and 2.55 times of those found in M. ruber M7. This work will make some contribution to the study of the biosynthesis pathway of MPs in M. ruber.

Author Contributions: L.L. designed and carried out the present research work, conducted experiments, analyzed the data and wrote the present manuscript. F.C. provided a place in the laboratory and gave access to the lab facilities for experimentation and funds for the present work. All authors have read and agreed to the published version of the manuscript.

Funding: This work was supported by the Major Program of the National Natural Science Foundation of China (Nos. 31730068 and 31330059 to F.C.), and the National Key Research and Development Program of China (No.2018YFD0400404 to F.C.).

Conflicts of Interest: The authors declare no conflict of interest. The funders had no role in the design of the study; in the collection, analyses, or interpretation of data; in the writing of the manuscript, or in the decision to publish the results.

\section{References}

1. Chen, W.P.; He, Y.; Zhou, Y.X.; Shao, Y.C.; Feng, Y.L.; Li, M.; Chen, F.S. Edible Filamentous Fungi from the Species Monascus: Early Traditional Fermentations, Modern Molecular Biology, and Future Genomics. Compr. Rev. Food Sci. Food Saf. 2015, 14, 555-567. [CrossRef]

2. Kim, S.C.; Lee, G.D.; Choi, I.H. Breast meat quality of broilers fed fermented red ginseng marc powder mixed with red-koji during storage. Emir. J. Food Agric. 2016, 28, 283-287. [CrossRef]

3. Feng, Y.L.; Shao, Y.C.; Chen, F.S. Monascus pigments. Appl. Microbiol. Biotechnol. 2012, 96, 1421-1440. [CrossRef]

4. Patakova, P. Monascus secondary metabolites: Production and biological activity. J. Ind. Microbiol. Biotechnol. 2013, 40, 169-181. [CrossRef] [PubMed]

5. Liu, J.; Lei, M.; Zhou, Y.; Chen, F. A Comprehensive Analysis of the Small GTPases Ypt7 Involved in the Regulation of Fungal Development and Secondary Metabolism in Monascus ruber M7. Front. Microbiol. 2019, 10, 452. [CrossRef] [PubMed]

6. Blanc, P.J.; Laussac, J.P.; Lebars, J.; Lebars, P.; Loret, M.O.; Pareilleux, A.; Prome, D.; Prome, J.C.; Santerre, A.L.; Goma, G. Characterization of Monascidin-a from Monascus as Citrinin. Int. J. Food Microbiol. 1995, 27, $201-213$. [CrossRef]

7. Mukherjee, G.; Singh, S.K. Purification and characterization of a new red pigment from Monascus purpureus in submerged fermentation. Process Biochem. 2011, 46, 188-192. [CrossRef]

8. Chen, W.; Feng, Y.; Molnar, I.; Chen, F. Nature and nurture: Confluence of pathway determinism with metabolic and chemical serendipity diversifies Monascus azaphilone pigments. Nat. Prod. Rep. 2019, 36, 561-572. [CrossRef]

9. Chen, W.P.; Chen, R.; Liu, Q.P.; He, Y.; He, K.; Ding, X.L.; Kang, L.J.; Guo, X.X.; Xie, N.N.; Zhou, Y.X.; et al. Orange, red, yellow: Biosynthesis of azaphilone pigments in Monascus fungi. Chem. Sci. 2017, 8, 4917-4925. [CrossRef]

10. Balakrishnan, B.; Chandran, R.; Park, S.H.; Kwon, H.J. A New Protein Factor in the Product Formation of Non-Reducing Fungal Polyketide Synthase with a C-Terminus Reductive Domain. J. Microbiol. Biotechnol. 2015, 25, 1648-1652. [CrossRef]

11. Chen, F.S.; Hu, X.Q. Study on red fermented rice with high concentration of monacolin $\mathrm{K}$ and low concentration of citrinin. Int. J. Food Microbiol. 2005, 103, 331-337. [CrossRef]

12. He, Y.; Liu, Q.; Shao, Y.; Chen, F. Ku70 and ku80 null mutants improve the gene targeting frequency in Monascus ruber M7. Appl. Microbiol. Biotechnol. 2013, 97, 4965-4976. [CrossRef] [PubMed]

13. Lei, M.; Liu, J.; Fang, Y.; Shao, Y.; Li, L.; Yu, J.H.; Chen, F. Effects of Different G-Protein alpha-Subunits on Growth, Development and Secondary Metabolism of Monascus ruber M7. Front. Microbiol. 2019, 10, 1555. [CrossRef] [PubMed] 
14. Liu, Q.; Xie, N.; He, Y.; Wang, L.; Shao, Y.; Zhao, H.; Chen, F. MpigE, a gene involved in pigment biosynthesis in Monascus ruber M7. Appl. Microbiol. Biotechnol. 2014, 98, 285-296. [CrossRef] [PubMed]

15. Shao, Y.C.; Ding, Y.D.; Zhao, Y.; Yang, S.; Xie, B.J.; Chen, F.S. Characteristic analysis of transformants in T-DNA mutation library of Monascus ruber. World J. Microbiol. Biotechnol. 2009, 25, 989-995. [CrossRef]

16. Li, L.; He, L.; Lai, Y.; Shao, Y.; Chen, F. Cloning and functional analysis of the Gbeta gene Mgb1 and the Ggamma gene Mgg1 in Monascus ruber. J. Microbiol. 2014, 52, 35-43. [CrossRef]

17. Mapari, S.A.S.; Thrane, U.; Meyer, A.S. Fungal polyketide azaphilone pigments as future natural food colorants? Trends Biotechnol. 2010, 28, 300-307. [CrossRef]

18. Lee, C.L.; Wen, J.Y.; Hsu, Y.W.; Pan, T.M. The blood lipid regulation of Monascus-produced monascin and ankaflavin via the suppression of low-density lipoprotein cholesterol assembly and stimulation of apolipoprotein A1 expression in the liver. J. Microbiol. Immunol. Infect. 2018, 51, 27-37. [CrossRef]

19. Bachovchin, D.A.; Cravatt, B.F. The pharmacological landscape and therapeutic potential of serine hydrolases. Nat. Rev. Drug Discov. 2012, 11, 52-68. [CrossRef]

20. Holmquist, M. Alpha/Beta-Hydrolase Fold Enzymes: Structures, Functions and Mechanisms. Curr. Protein Pept. Sci. 2000, 1, 209-235. [CrossRef]

21. Bun, J.S.; Slack, M.D.; Schemenauer, D.E.; Johnson, R.J. Comparative analysis of the human serine hydrolase OVCA2 to the model serine hydrolase homolog FSH1 from S. cerevisiae. PLoS ONE 2020, 15, e0230166. [CrossRef] [PubMed]

22. Long, J.Z.; Cravatt, B.F. The Metabolic Serine Hydrolases and Their Functions in Mammalian Physiology and Disease. Chem. Rev. 2011, 111, 6022-6063. [CrossRef] [PubMed]

23. Navia-Paldanius, D.; Savinainen, J.R.; Laitinen, J.T. Biochemical and pharmacological characterization of human alpha/beta-hydrolase domain containing 6 (ABHD6) and 12 (ABHD12). J. Lipid Res. 2012, 53, $2413-2424$. [CrossRef] [PubMed]

24. Ritchie, M.K.; Johnson, L.C.; Clodfelter, J.E.; Pemble, C.W.; Fulp, B.E.; Furdui, C.M.; Kridel, S.J.; Lowther, W.T. Crystal Structure and Substrate Specificity of Human Thioesterase 2: Insights into the Molecular Basis for the Modulation of Fatty Acid Synthase. J. Biol. Chem. 2016, 291, 3520-3530. [CrossRef] [PubMed]

25. Liu, J.; Zhou, Y.; Yi, T.; Zhao, M.; Xie, N.; Lei, M.; Liu, Q.; Shao, Y.; Chen, F. Identification and role analysis of an intermediate produced by a polygenic mutant of Monascus pigments cluster in Monascus ruber M7. Appl. Microbiol. Biotechnol. 2016, 100, 7037-7049. [CrossRef]

26. Fisch, K.M.; Skellam, E.; Ivison, D.; Cox, R.J.; Bailey, A.M.; Lazarus, C.M.; Simpson, T.J. Catalytic role of the C-terminal domains of a fungal non-reducing polyketide synthase. Chem. Commun. 2010, 46, 5331-5333. [CrossRef] [PubMed]

(C) 2020 by the authors. Licensee MDPI, Basel, Switzerland. This article is an open access article distributed under the terms and conditions of the Creative Commons Attribution (CC BY) license (http://creativecommons.org/licenses/by/4.0/). 